\title{
Microstructured optical fiber Bragg grating-based shear stress sensing in adhesive bonds
}

Sulejmani, Sanne, Sonnenfeld, Camille, Geernaert, Thomas, Luyckx, Geert, Van Hemelrijck, Danny, et al.

Sanne Sulejmani, Camille Sonnenfeld, Thomas Geernaert, Geert Luyckx, Danny Van Hemelrijck, Pawel Mergo, Krzysztof Skorupski, Waclaw Urbanczyk, Karima Chah, Hugo Thienpont, Francis Berghmans, "Microstructured optical fiber Bragg grating-based shear stress sensing in adhesive bonds," Proc. SPIE 9157, 23rd International Conference on Optical Fibre Sensors, 91577P (2 June 2014); doi: 10.1117/12.2059076

SPIE Event: OFS2014 23rd International Conference on Optical Fiber Sensors, 2014, Santander, Spain 


\title{
Microstructured optical fiber Bragg grating-based shear stress sensing in adhesive bonds
}

\author{
Sanne Sulejmani ${ }^{\mathrm{a}^{*}}$, Camille Sonnenfeld ${ }^{\mathrm{a}}$, Thomas Geernaert ${ }^{\mathrm{a}}$, Geert Luyckx ${ }^{\mathrm{b}}$, Danny Van \\ Hemelrijck $^{\mathrm{c}}$, Pawel Mergo ${ }^{\mathrm{d}}$, Krzysztof Skorupski ${ }^{\mathrm{d}}$, Waclaw Urbanczyk ${ }^{\mathrm{e}}$, Karima Chah ${ }^{\mathrm{f}}$, Hugo \\ Thienpont ${ }^{\mathrm{a}}$, Francis Berghmans ${ }^{\mathrm{a}}$ \\ ${ }^{a}$ Brussels Photonics Team, Vrije Universiteit Brussel, Pleinlaan 2, 1050 Brussel, Belgium; ${ }^{b}$ Dept. of \\ Materials Science and Engineering, Universiteit Gent, Technologiepark 903, 9052 Gent, Belgium; \\ ${ }^{c}$ Dept. of Mechanics of Materials and Constructions, Vrije Universiteit Brussel, Pleinlaan 2, 1050 \\ Brussel, Belgium; ${ }^{\mathrm{d} D e p t . ~ o f ~ O p t i c a l ~ F i b e r ~ T e c h n o l o g y, ~ M a r i e ~ C u r i e-S k l o d o w s k a ~ U n i v e r s i t y, ~ P l . ~ M . ~}$ \\ Curie-Sklodowskiej 3, 20-031 Lublin, Poland; 'Institute of Physics, Wroclaw University of \\ Technology, Wybrzeze Wyspianskiego 27, 50-370 Wroclaw, Poland; ${ }^{\mathrm{f}}$ Electromagnetism and \\ Telecom Dept., University of Mons, Mons 7000, Belgium
}

\begin{abstract}
We present shear stress sensing with a Bragg grating sensor fabricated in a highly birefringent microstructured optical fiber. This sensor has a shear strain sensing resolution of $0.04 \mathrm{pm} / \mu \varepsilon$ when embedded in a shear loaded adhesive bond. We achieve discrete shear stress mapping in an adhesive bond by embedding a multitude of these sensors at different locations in the bond line. Experiments and numerical modeling show the limited influence of angular misalignment of the sensor on its shear stress response. Finally, we discuss the cross-sensitivity of this sensor to shear strain and temperature.
\end{abstract}

Keywords: microstructured optical fiber, fiber Bragg grating, sensor, shear strain, structural health monitoring

\section{INTRODUCTION}

Fiber Bragg grating (FBG) based sensors are studied intensively for strain monitoring applications, for example for structural health monitoring (SHM) purposes. This type of sensor offers well-known advantages, including low weight, multiplexing capabilities, immunity to electromagnetic interference and the ability to be embedded in a non-intrusive manner in materials and structures [1].

Axial strain sensing with FBG sensors has become a successful alternative to electrical strain gauges. In recent years, promising results have also been reported on FBG sensors fabricated in highly birefringent fibers for transverse strain sensing [2]. However, there are still important drawbacks involved in transverse strain sensing with conventional polarization maintaining fibers. High birefringence in these fibers is induced by stress applying parts in the fiber cladding, which are sections of materials with different thermal expansion properties than the non-doped silica cladding. This results in a significant thermal cross-sensitivity of these sensors, while their transverse strain sensing resolution is low. Microstructured optical fibers (MOFs) have been proposed to overcome this cross-sensitivity. High birefringence in these fibers is induced by an asymmetric air hole geometry and only a small and low $\mathrm{GeO}_{2}$-doped region is needed to allow for Bragg grating inscription using conventional UV inscription techniques. Moreover, their transverse strain sensitivity can be enhanced by properly tuning the air hole geometry, i.e. by altering the number of air holes, their sizes and/or their positions. Results of the 'butterfly' microstructured optical fiber Bragg grating (MOFBG) sensor demonstrate that by designing dedicated MOFs one can improve the transverse strain sensitivity by an order of magnitude compared to conventional polarization maintaining fibers [3].

Besides the ability to sense the normal strains mentioned above, shear strain sensing is also important for SHM purposes. Excessive shear strain could for example lead to disbonding of adhesive bonds or to delamination in composite materials. Continuous assessing shear stress could therefore help to prevent catastrophic failure of structures. Fiber optic shear stress sensing has received fairly little attention so far. Recently we have shown that the butterfly MOFBG sensor can be 
used as well for shear strain sensing. By aligning the optical axes parallel with the directions of principal strain in a shear loaded material, the butterfly MOFBG sensor will detect the shear load induced transverse strain [4].

In this work, we embed 3 butterfly MOFBG sensors in an adhesive bond to map the shear stress distribution along the overlap of a single lap adhesive joint (SLJ). Because of the asymmetric air hole geometry of the butterfly MOF, angular misalignment will influence the shear stress sensitivity. We investigate, experimentally and with finite element modeling (FEM), the influence of angular alignment when the sensor is embedded in a SLJ. Finally, we discuss the crosssensitivity of the butterfly MOFBG shear stress sensor to temperature.

\section{ANGULAR ALIGNMENT DEPENDENCE OF MOFBG-BASED SHEAR STRESS SENSOR}

The sensing principle of the butterfly MOFBG sensor relies on the change of modal birefringence $B_{\text {mod }}$ caused by a mechanical load that is applied to the cladding of the fiber, or to the host material in which the sensor is embedded [5]. Because of the asymmetric microstructure (Figure 1), a radial load will induce an asymmetric mechanical stress distribution in the core of the fiber. The refractive indices $n_{x}$ and $n_{y}$ of the modes polarized along the $x$ - and $y$-direction are therefore affected in a different manner. This is translated into a change of the separation $\Delta \lambda$ between the individual Bragg peak wavelengths $\lambda_{i}$ for each linearly polarized mode, which is directly linked to $B_{\text {mod }}$ as given by $\Delta \lambda=2 \cdot B_{\text {mod }} \Lambda$, with $\Lambda$ the period of the FBG. We consider $\Delta \lambda$ as the sensor signal, and the sensitivity of the butterfly MOFBG sensor corresponds to the rate of change of $\Delta \lambda$ when submitted to a specific mechanical or thermal load. The butterfly MOFBG sensor has a sine-like dependence of the transverse strain sensitivity to the angular orientation of the microstructure with respect to the direction of the applied load [6]. It is most sensitive when the load is applied along $\alpha=90^{\circ}$, which is indicated by the slow-axis or $y$ '-axis in Figure 1. However, when the sensor is embedded in a shear loaded material at an angular orientation $\alpha=+45^{\circ}$ or $\alpha=-45^{\circ}$, it will detect the transverse strain that is induced by the shear load. By orienting the sensor at $+45^{\circ}$ or $-45^{\circ}$, the fundamental optical axes are aligned parallel to the directions of principal strain in the material.

We have already demonstrated the shear stress sensing functionality of the butterfly MOFBG sensor by embedding it in the adhesive of a single lap adhesive joint (SLJ). When this joint is tensile loaded, the adhesive and the embedded fiber sensor will be shear loaded. We neglect the small amount of peel stress that is also present in the adhesive, because the peel stress level is only significant close to the edges of the overlap when compared to the shear stress level. The twodimensional linear elastic analysis method of Goland-Reissner describes the shear stress distribution along the length of the adhesive overlap [7]. Experiments and 2D finite element modeling have shown that the shear stress sensitivity of the butterfly MOFBG sensor, when angularly rotated at $-45^{\circ}$ and embedded centrally in the adhesive layer, is about 60 $\mathrm{pm} / \mathrm{MPa}[4]$.

In this paper we embed 3 butterfly MOFBG sensors at different locations in an adhesive bond line to investigate their potential for distributed shear stress mapping. The shear stress sensors are oriented as closely as possible to an angle of $45^{\circ}$ in order to be maximally sensitive to shear load and they are located where peel stress is much lower than the shear stress for only the shear stress to be picked up by the sensors. The length of the overlap is $25.6 \mathrm{~mm}$ and the 3 sensors are positioned respectively at $\mathrm{x}_{1}=-10.0 \mathrm{~mm}, \mathrm{x}_{2}=0.3 \mathrm{~mm}$, and $\mathrm{x}_{3}=10.4 \mathrm{~mm}$ (Figure 1). The two-component epoxy adhesive bond has a thickness of $0.7 \mathrm{~mm}$ and width of $25.6 \mathrm{~mm}$. The sample is tensile loaded up to a maximum of $2.5 \mathrm{kN}$. The responses of the embedded sensors are monitored using a static FBG interrogator (FBG scan 608) with a peak wavelength detection accuracy of $1 \mathrm{pm}$ and a sample frequency of $1 \mathrm{~Hz}$ per channel. To derive the shear stress sensitivity of the sensors we first determine their sensitivity to tensile loading by a linear regression analysis of the FBG sensor responses. Sensor 1 has a response of $109.9 \mathrm{pm} / \mathrm{kN}$, sensor 2 has a response of $51.1 \mathrm{pm} / \mathrm{kN}$ and sensor 3 has a response of $141.5 \mathrm{pm} / \mathrm{kN}$. Next, we use the analytical model of Goland-Reissner to calculate the shear stress at the position of the fiber sensor. We achieve shear stress sensitivities of $51.3 \mathrm{pm} / \mathrm{MPa}, 63.3 \mathrm{pm} / \mathrm{MPa}$ and $60.5 \mathrm{pm} / \mathrm{MPa}$ for sensor 1 , sensor 2 and sensor 3, respectively.

A similar sample is analyzed with two dimensional FEM, using CAE Abaqus Software. We model the change in mechanical stress distribution in the core region of the fibers due to tensile loading of the joint. From this stress distribution and the stress-optic coefficients, we can calculate the induced change in refractive indices, and hence the change in Bragg peak wavelengths and their separation $\Delta \lambda$ [4]. Our FEM simulations result in shear stress sensitivities of $59.4 \mathrm{pm} / \mathrm{MPa}, 62.3 \mathrm{pm} / \mathrm{MPa}$ and $63.3 \mathrm{pm} / \mathrm{MPa}$, for respectively sensor 1, sensor 2 and sensor 3, which corresponds well to our experimental values. 
These shear stress sensitivities correspond to an average shear strain sensitivity of $0.04 \mathrm{pm} / \mu \varepsilon$, or a shear strain sensing resolution of $50 \mu \varepsilon$. In typical adhesive bond lines the level of shear stress is several millistrains. Hence, this resolution corresponds to $0.5-1 \%$ of the measurement range, which allows measuring shear stress with a high sensitivity at different locations in the adhesive.

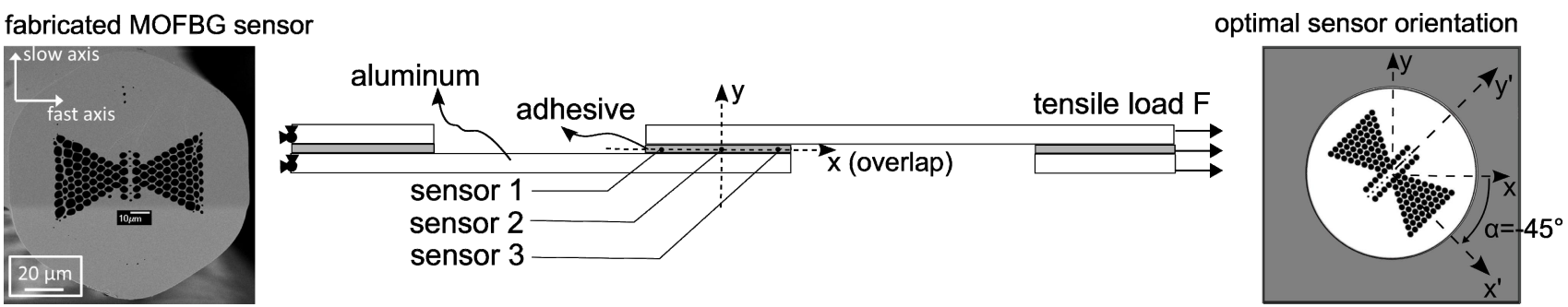

Figure 1. Configuration of a SLJ with 3 embedded butterfly MOFBG sensors at an angular orientation $\alpha=-45^{\circ}$. Tensile loading of the joint will induce shear loading of the adhesive layer. A scanning electron micrograph (SEM) of a fabricated butterfly MOFBG sensor is also shown.

The butterfly MOFBG sensor has an asymmetric geometry, which implies that its response to radially applied load strongly depends on the angular orientation of the fiber microstructure with respect to the direction of the load. An angular misalignment of the embedded sensor from the optimal orientation at $+45^{\circ}$ or $-45^{\circ}$, could lead to an underestimation of the shear stress level and an increased cross-sensitivity to peel or transverse stress. Although extensive research has been performed on radial load sensing with this sensor, fixing the orientation of this type of highly birefringent fiber remains a challenge. After testing of the sample, the sides were polished to determine the actual fiber sensor orientations to be $\alpha_{1}=-50.9^{\circ}, \alpha_{2}=-47.3^{\circ}$, and $\alpha_{3}=-52.5^{\circ}$, leading to an angular misalignment of maximum $8^{\circ}$. To investigate the influence of this misalignment on the shear stress sensitivity, we perform experiments and 2D FEM modeling of sensors embedded at multiple orientations. Six samples, with in each sample 3 embedded sensors, are experimentally tested. The results are shown in Figure 2. The sensor has a maximum sensitivity to shear stress when embedded at an orientation of $+45^{\circ}$ or $-45^{\circ}$, i.e. when the optical axes are aligned parallel to the axes of principal strain in the material. The sensitivity decays at fiber orientations of $0^{\circ},-90^{\circ}$ or $+90^{\circ}$, which are optimal directions to measure peel or transverse load. 2D FEM simulations of the response of sensors at different orientations, lead to similar results. This research demonstrates that a misalignment of $8^{\circ}$ leads to a shear stress sensitivity decrease of only $6 \%$.

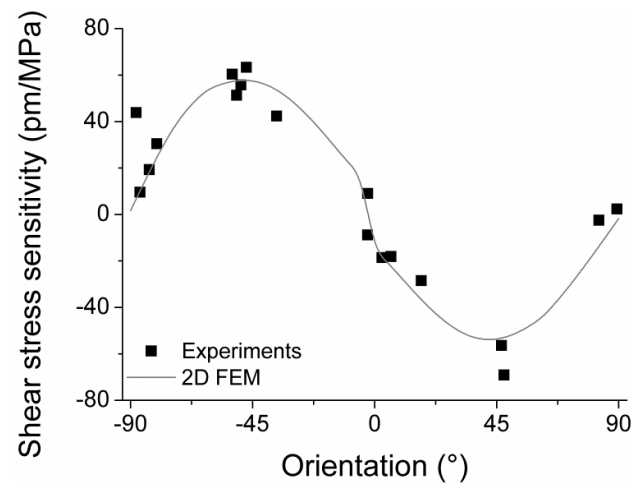

Figure 2. The experimental (scattered points) and numerically modeled (solid line) shear stress sensitivity of a butterfly MOFBG sensor embedded in the adhesive layer of a SLJ at different angular orientations. The optimal orientation for shear stress sensing is $+45^{\circ}$ or $-45^{\circ}$, when the fundamental optical axes are aligned parallel to the directions of principal strain in the material.

\section{THERMAL CROSS-SENSITIVITY OF THE MOFBG-BASED SHEAR STRESS SENSOR}

An important advantage of highly birefringent MOFBG-based strain sensors is that their modal birefringence is not strongly influenced by temperature fluctuations. The only contribution to the thermal sensitivity of a bare fiber stems 
from the $\mathrm{GeO}_{2}$-doped region in the (asymmetric) core region. We have previously demonstrated that the thermal sensitivity of the Bragg peak separation is only $-0.1 \mathrm{pm} /{ }^{\circ} \mathrm{C}[6]$. However, once this fiber is embedded for strain monitoring applications, the thermal induced mechanical stress in the host material will affect $B_{m o d}$ and the sensor signal. This cross-sensitivity to strain and temperature can be important and should not be neglected.

We experimentally investigate the thermal cross-sensitivity of the shear strain sensitivity when the sensor is embedded in a single lap adhesive joint. A SLJ sample is fabricated with a butterfly MOFBG sensor embedded in the centre with an angular orientation of $-42^{\circ}$. The sample is subjected to a tensile load of maximum $3 \mathrm{kN}$. The test is repeated at $20^{\circ} \mathrm{C}$, at $35^{\circ} \mathrm{C}$ and $50^{\circ} \mathrm{C}$, and the shear stress sensitivity is determined at each temperature. A temperature of $50^{\circ} \mathrm{C}$ is at the upper limit of the usable range of the adhesive. We find that the shear stress sensitivity increases by $8 \%$ when the test is performed at $35^{\circ} \mathrm{C}$, and that it increases again by $30 \%$ when the test is performed at $50^{\circ} \mathrm{C}$. These results demonstrate that small temperature fluctuations will have a small influence on the shear stress measurements. The strong increase in sensor response at $50^{\circ} \mathrm{C}$ can be attributed to the decreasing adhesive performance. These results also demonstrate that, although the bare butterfly MOFBG sensor intrinsically operates in a temperature insensitive manner, thermal crosssensitivity can have an important influence on strain measurements once the sensor is embedded. Therefore, when temperature insensitive shear strain sensing is targeted, one should either use an additional temperature compensation technique, or tune the design of the MOFBG sensor as such that it features a negligible thermal sensitivity once embedded in a specific structure.

\section{CONCLUSION}

We have demonstrated, both with experiments and with 2D FEM simulations, that we can measure the shear stress distribution in the adhesive bond line of a single lap joint by embedding several butterfly MOFBG sensors at different locations along the overlap. These shear sensors have a shear strain sensing resolution of $50 \mu \varepsilon$, which corresponds to 0.5 $-1 \%$ of the measurement range in typical adhesive bonds. Moreover, we have shown that a small angular misalignment has a limited influence of the shear stress sensitivity: a misalignment of $8^{\circ}$ leads to a sensitivity decrease of $6 \%$. Finally, we have investigated the thermal cross-sensitivity of the shear stress sensitivity and we can conclude that small temperature fluctuations will have a limited influence on the shear stress measurements.

\section{REFERENCES}

[1] G. Luyckx, E. Voet, N. Lammens, and J. Degrieck, "Strain Measurements of Composite Laminates with Embedded Fibre Bragg Gratings: Criticism and Opportunities for Research," Sensors 11, 384-408 (2010).

[2] G. Luyckx, E. Voet, T. Geernaert, K. Chah, T. Nasilowski, W. De Waele, W. Van Paepegem, M. Becker, H. Bartelt, W. Urbanczyk, J. Wojcik, J. Degrieck, F. Berghmans, and H. Thienpont, "Response of FBGs in Microstructured and Bow Tie Fibers Embedded in Laminated Composite," IEEE Photonics Technol. Lett. 21, 1290-1292 (2009).

[3] C. Sonnenfeld, S. Sulejmani, T. Geernaert, S. Eve, N. Lammens, G. Luyckx, E. Voet, J. Degrieck, W. Urbanczyk, P. Mergo, M. Becker, H. Bartelt, F. Berghmans, and H. Thienpont, "Microstructured Optical Fiber Sensors Embedded in a Laminate Composite for Smart Material Applications," Sensors 11, 2566-2579 (2011).

[4] S. Sulejmani, C. Sonnenfeld, T. Geernaert, G. Luyckx, D. Van Hemelrijck, P. Mergo, W. Urbanczyk, K. Chah, C. Caucheteur, P. Megret, H. Thienpont, F. Berghmans, "Shear stress sensing with Bragg grating-based sensors in microstructured optical fibers," Optics Express 21, 20404-20416 (2013).

[5] T. Martynkien, G. Statkiewicz-Barabach, J. Olszewski, J. Wojcik, P. Mergo, T. Geernaert, C. Sonnenfeld, A. Anuszkiewicz, M. K. Szczurowski, K. Tarnowski, M. Makara, K. Skorupski, J. Klimek, K. Poturaj, W. Urbanczyk, T. Nasilowski, F. Berghmans, and H. Thienpont, "Highly birefringent microstructured fibers with enhanced sensitivity to hydrostatic pressure," Opt. Express 18, 15113-15121 (2010).

[6] S. Sulejmani, C. Sonnenfeld, T. Geernaert, F. Berghmans, H. Thienpont, S. Eve, N. Lammens, G. Luyckx, E. Voet, J. Degrieck, W. Urbanczyk, P. Mergo, M. Becker, and H. Bartelt, "Towards micro-structured optical fiber sensors for transverse strain sensing in smart composite materials," in IEEE Sensors 2011, pp. 109-112 (2011).

[7] L.F.M. Da Silva, P.J.C. das Neves, R.D. Adams, J.K. Spelt, "Analytical models of adhesively bonded jointsPart I: Literature survey,” International Journal of Adhesion and Adhesives 29 (3), 319-330 (2009). 